\title{
Effect of high dose folic acid supplementation in pregnancy on pre-eclampsia (FACT): double blind, phase III, randomised controlled, international, multicentre trial
}

\author{
Shi Wu Wen, ${ }^{1,2,3}$ Ruth Rennicks White, ${ }^{1}$ Natalie Rybak, ${ }^{1}$ Laura M Gaudet, ${ }^{1,2,3}$ \\ Stephen Robson, ${ }^{4}$ William Hague, ${ }^{5,6}$ Donnette Simms-Stewart, ${ }^{7}$ Guillermo Carroli, ${ }^{8}$ \\ Graeme Smith, ${ }^{9}$ William D Fraser, ${ }^{10,11,12}$ George Wells, ${ }^{2,13,14,15}$ Sandra T Davidge, ${ }^{16,17,18}$ \\ John Kingdom, ${ }^{19}$ Doug Coyle, ${ }^{2}$ Dean Fergusson, ${ }^{15,20}$ Daniel J Corsi, ${ }^{1}$ Josee Champagne, ${ }^{15}$ \\ Elham Sabri, ${ }^{15}$ Tim Ramsay, ${ }^{15,20}$ Ben Willem J Mol, ${ }^{5,21}$ Martijn A Oudijk, ${ }^{22,23}$ Mark C Walker ${ }^{1,23}$
} For numbered affiliations see
end of article.

Correspondence to: MC Walker, Ottawa Hospital Research Institute, Ottawa, ON, K1H 8L6, Canada mwalker@ohri.ca Additional material is published online only. To view please visit the journal online.

Cite this as: $B M J$ 2018;362:k3478 http://dx.doi.org/10.1136/bmj.k3478

Accepted: 11 July 2018

\section{ABSTRACT}

OBJECTIVE

To determine the efficacy of high dose folic acid supplementation for prevention of pre-eclampsia in women with at least one risk factor: pre-existing hypertension, prepregnancy diabetes (type 1 or 2), twin pregnancy, pre-eclampsia in a previous pregnancy, or body mass index $\geq 35$.

\section{DESIGN}

Randomised, phase III, double blinded international, multicentre clinical trial.

SETTING

70 obstetrical centres in five countries (Argentina, Australia, Canada, Jamaica, and UK).

PARTICIPANTS

2464 pregnant women with at least one high risk factor for pre-eclampsia were randomised between 2011 and 2015 (1144 to the folic acid group and 1157 to the placebo group); 2301 were included in the intention to treat analyses.

INTERVENTION

Eligible women were randomised to receive either daily high dose folic acid (four $1.0 \mathrm{mg}$ oral tablets) or placebo from eight weeks of gestation to the end of week 16 of gestation until delivery. Clinicians, participants, adjudicators, and study staff were masked to study treatment allocation.

MAIN OUTCOME MEASURE

The primary outcome was pre-eclampsia, defined as hypertension presenting after 20 weeks'

\section{WHAT IS ALREADY KNOWN ON THIS TOPIC}

Evidence from epidemiological and biological studies has shown a clear doseresponse relation between increasing folic acid supplementation and decreasing risk of pre-eclampsia in women with additional identified risk factors

Until now, a lack of randomised evidence has limited the development of a comprehensive recommendation for the use of high dose folic acid for prevention of pre-eclampsia in women at high risk of developing pre-eclampsia

\section{WHAT THIS STUDY ADDS}

This study suggests that high dose folic acid supplementation in later pregnancy has no benefit for preventing pre-eclampsia

However, folic acid supplementation remains indicated in preconception and early pregnancy but there is a need to define when to discontinue supplementation as current clinical practice guidelines do not provide clear guidance beyond the first trimester gestation with major proteinuria or HELLP syndrome (haemolysis, elevated liver enzymes, low platelets).

\section{RESULTS}

Pre-eclampsia occurred in 169/1144 (14.8\%) women in the folic acid group and 156/1157 (13.5\%) in the placebo group (relative risk 1.10, 95\% confidence interval 0.90 to $1.34 ; \mathrm{P}=0.37$ ). There was no evidence of differences between the groups for any other adverse maternal or neonatal outcomes.

\section{CONCLUSION}

Supplementation with $4.0 \mathrm{mg} /$ day folic acid beyond the first trimester does not prevent pre-eclampsia in women at high risk for this condition.

TRIAL REGISTRATION

Current Controlled Trials ISRCTN23781770 and

ClinicalTrials.gov NCT01355159.

\section{Introduction}

Pre-eclampsia is a serious medical condition, affecting about $3-5 \%$ of pregnancies, ${ }^{12}$ accounting for more than 35000 maternal deaths annually worldwide and an important factor in maternal morbidity. ${ }^{134}$ Preeclampsia affects multiple organ systems and leads to an increased risk of severe complications in pregnancy. ${ }^{5}$ Since delivery of the placenta is the only known cure, pre-eclampsia is a leading cause of indicated preterm delivery, ${ }^{12}$ perinatal morbidity, mortality, and long term disability. ${ }^{67}$ Epidemiological studies of the association between folic acid supplementation and the incidence of pre-eclampsia have shown a potential protective effect, ${ }^{9}$ although findings have been inconsistent. ${ }^{8-14}$ In a randomised trial of supplementation with a multivitamin containing $0.8 \mathrm{mg}$ folic acid and hypertension in pregnancy in a high risk population of women positive for antibodies to HIV, a 38\% reduction was observed in the primary composite outcome of gestational hypertension (including pre-eclampsia or eclampsia) in the intervention group compared with placebo group. ${ }^{15}$ Other forms of folate, including 5-methyltetrahydrofolate, have been investigated with similar results, ${ }^{16}$ whereas folic acid antagonists have shown the opposite effect, increasing the risk of preeclampsia. $^{17}$

Based on large randomised trials, ${ }^{1819}$ supplementation of folic acid to prevent neural tube defects has been recommended worldwide during the preconception period and the first trimester of pregnancy. Recommended 
doses are 4.0-5.0 mg daily up to 12 weeks' gestation for women at high risk of having an affected fetus, and 0.4-1.0 mg daily for women at low risk. ${ }^{20-22}$ Although the neural tube closes in the first trimester, preeclampsia is a two stage disorder, with the first stage occurring in the late first trimester (after eight weeks) and the second stage occurring in the third trimester. Supplementation of high doses of folic acid in early gestation may work at both stages of pre-eclampsia development, and a larger dose in the late first or early second trimester (between eight and 16 week's gestation) during the peak period of placental growth and development may be most effective in preventing pre-eclampsia. Findings from the Ottawa and Kingston (OaK) Birth Cohort suggested a 60\% reduction in risk of pre-eclampsia and a dose-response association between folic acid and risk of pre-eclampsia in women with identified risk factors. ${ }^{923}$ A high daily dose $(4.0 \mathrm{mg})$ of folic acid might be needed for these women because they may have placental, endothelial, and metabolic defects (including those of folate metabolism) leading to an increased risk of developing pre-eclampsia. Previous studies have been observational in nature and thus warranted a large randomised controlled trial. The current study, the Folic Acid Clinical Trial (FACT), was designed and conducted to evaluate the effect of daily supplementation with $4.0 \mathrm{mg}$ folic acid beyond the first trimester on the risk of developing preeclampsia among pregnant women at high risk for this condition.

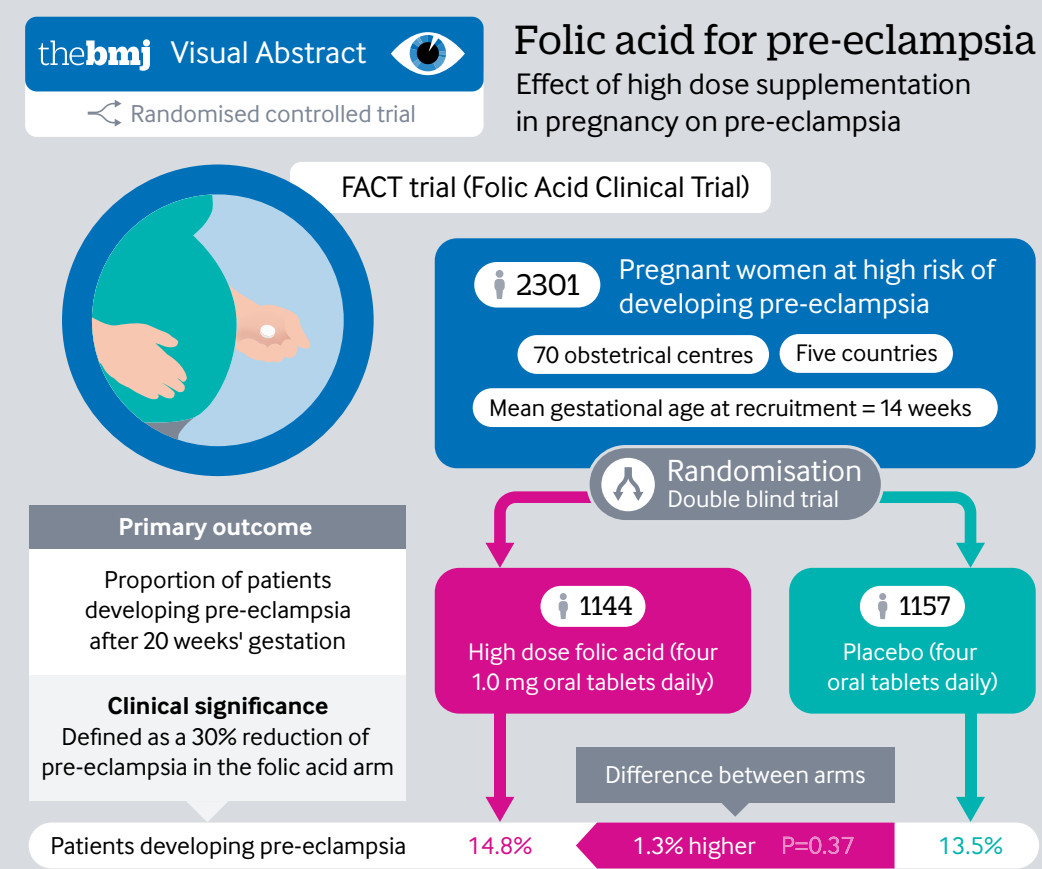

No benefit found for high dose folic acid supplementation beyond the first trimester for the prevention of pre-eclampsia or related maternal and neonatal adverse outcomes.

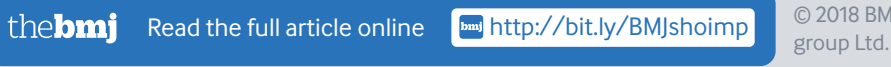

\section{Methods}

Trial design and study population

FACT was a randomised, double blinded, placebo controlled, phase III, international multicentre trial carried out at 70 high risk obstetric referral centres covering diverse populations in Canada, Argentina, Australia, Jamaica, and the United Kingdom (see supplementary table S1).

We considered pregnant women to be eligible for participation in the trial if they were between eight and 16 completed weeks of gestation with a confirmed viable fetus and at least one of the following risk factors for pre-eclampsia: pre-existing hypertension, prepregnancy diabetes (type 1 or 2), twin pregnancy, pre-eclampsia in a previous pregnancy, or body mass index (BMI) $\geq 35 \mathrm{~kg} / \mathrm{m}^{2}$. Documentation of BMI measured (height and weight) between three months before pregnancy and up to the time of randomisation was required as part of study eligibility. We excluded women if they had a known fetal anomaly or fetal death, a history of maternal medical complications (including renal disease with altered renal function), epilepsy, cancer, or current use of folic acid antagonists, illicit drug or alcohol misuse ( $\geq 2$ drinks daily) during current pregnancy, known hypersensitivity to folic acid, multiple pregnancy, previous participation in this trial, or a history or presence of any important disease or condition that would preclude the use of high dose (up to $5.1 \mathrm{mg}$ daily) folic acid.

\section{Participant recruitment and randomisation}

The purpose and requirements of the trial were explained to eligible women, and after written informed consent, we randomised participants to either folic acid or placebo.

The Methods Centre at Ottawa Hospital Research Institute implemented randomisation using a web based randomisation platform that generated a unique randomisation ID. Randomisation used variable permuted blocks of four and six with stratification by centre. Study data were entered into the web based platform at each site. Study treatment was centrally prepared, pre-labelled with unique study IDs and provided to each site.

The trial intervention consisted of $4.0 \mathrm{mg}$ folic acid or placebo, taken as four $1.0 \mathrm{mg}$ tablets once daily, from randomisation (8-16 completed weeks of gestation) until delivery. Participants could continue taking prenatal vitamins or low dose folic acid supplements containing up to $1.1 \mathrm{mg}$ of folic acid. The folic acid and placebo tablets had no taste and an identical external appearance, thereby masking participants to their treatment group. The data coordinating centre managed treatment allocation, and all participants, site investigators, coordinators and other research staff, and members of the trial coordinating centre were blinded to treatment allocation after randomisation. No unmasking occurred during the trial. 
Frequency and duration of follow-up

A total of four follow-up visits occurred at 24-26 completed weeks of gestation, 34-36 completed weeks of gestation, after delivery, and 42 days post partum. At the initial study visit, information was collected on personal characteristics and maternal medical history. At study visits we carried out a physical examination (blood pressure, weight, urinalysis (urine dipstick), and fetal wellbeing) and documented concomitant drugs. Laboratory values were obtained at delivery, and maternal and neonatal information were collected from hospital records. At each visit we assessed and documented adverse events. Adherence to the study treatment was determined with the aid of a drug diary and pill counts. We asked participants to return their study treatment bottles at each visit. Compliance was measured as the percentage of pills remaining in the returned bottles, and participants completed the Dietary Folate Equivalent Screener (Block Food Frequency Questionnaire: www.nutritionquest.com) at randomisation and at 24-26 completed weeks of gestation.

\section{Primary outcome}

The primary outcome was pre-eclampsia, defined using the accepted definition at the time the trial commenced: diastolic blood pressure $\geq 90 \mathrm{~mm} \mathrm{Hg}$ on two occasions four hours or more apart and proteinuria (more than ++ on dipstick, or urinary protein $\geq 300 \mathrm{mg}$ in 24 hour urine collection, or random protein:creatinine ratio $\geq 30 \mathrm{mg}$ protein $/ \mathrm{mmol}$ ) in women at 20 weeks of gestation or greater, or diagnosis of HELLP syndrome (haemolysis, elevated liver enzymes, low platelets) or superimposed pre-eclampsia (history of pre-existing hypertension diagnosed before pregnancy or before 20 weeks' gestation with new proteinuria). ${ }^{2}$ The primary outcome (the trial protocol definition of pre-eclampsia) was adjudicated based on the consensus opinion of three investigators (MW, LG, and SWW). Adjudication was conducted before any statistical data analysis, masked to treatment group, country, and site. We excluded women from the primary outcome analysis who experienced a miscarriage, experienced early intrauterine fetal death (20-24 weeks of gestation), or withdrew consent.

\section{Secondary outcomes}

Prespecified secondary outcomes included maternal death, severe pre-eclampsia (pre-eclampsia with convulsion or HELLP or delivery $<34$ completed weeks of gestation), placental abruption, preterm delivery (<37 completed weeks of gestation), premature rupture of membranes, antenatal inpatient length of stay, intrauterine growth restriction $(<3 \mathrm{rd}$ centile), perinatal mortality, spontaneous abortion (miscarriage), stillbirth, neonatal mortality, neonatal morbidity (retinopathy of prematurity, periventricular leukomalacia, early onset sepsis, necrotising enterocolitis, intraventricular haemorrhage, ventilation, need for oxygen at 28 days), and length of stay in neonatal intensive care unit.
Adverse events reporting and safety monitoring

An independent data safety and monitoring board oversaw the safety of the trial. We collected and reviewed all adverse events from randomisation to 42 days post partum. An adverse event was defined as an untoward medical occurrence in a participant that may or may not have had a causal relation to the study treatment. A serious adverse event was defined as any untoward medical occurrence that resulted in maternal, fetal, or neonatal death; was life threatening; required prolonged hospital stay; caused persistent or major disability, incapacity, or congenital anomaly; or was deemed an important medical event. No safety issues were detected after the independent data safety and monitoring board's review.

\section{Statistical analysis}

On the basis of data from high risk pregnant women, ${ }^{24}$ we originally estimated that a sample of 3656 pregnant women was needed to detect a $30 \%$ reduction of preeclampsia from $12.0 \%$ to $8.4 \%$ (90\% power, two sided type I error 0.05) and to allow for up to $30 \%$ non-adherence, withdrawal, loss to follow-up, or other unanticipated events. However, owing to budget limitations, and because our follow-up rate was better than expected, we recalculated the sample size to 2464 women, which retained a study power of greater than $80 \%$ and still allowed for up to $10 \%$ loss to follow-up and withdrawal. In September 2015 the trial steering committee approved the sample size adjustment, and we notified the independent data safety and monitoring board. Based on the recruitment rate at the time of these meetings, a total new anticipated recruitment target of 2464 participants would be achieved by November, and as a result no interim analysis of outcomes would be conducted.

The analysis was carried out on an intention to treat basis. We first compared the baseline characteristics, compliance, folic acid intake from other sources, and supplementation with aspirin or calcium, or both, between intervention and placebo groups. The outcomes between these groups were then compared, using $\chi^{2}$ tests for the incidence of pre-eclampsia and categorical secondary outcomes and $t$ tests for the means of continuously distributed secondary outcomes. Multiple log binomial regression was conducted on the primary outcome to adjust for potential confounding by parity, age, cigarette smoking, and other important prognostic factors identified a priori. A generalised estimating equation model was used to account for the correlation between two fetuses or infants from the same pregnancy in analyses of neonatal outcomes. Treatment effects are expressed as relative risk with 95\% confidence intervals. No allowance for multiplicity was made for secondary outcomes. All statistical analyses were performed using Statistical Analysis System, version 9.1 (SAS Institute, Cary, NC).

\section{Patient involvement}

Although we did not actively seek patient engagement in the development of this protocol, physicians' input 


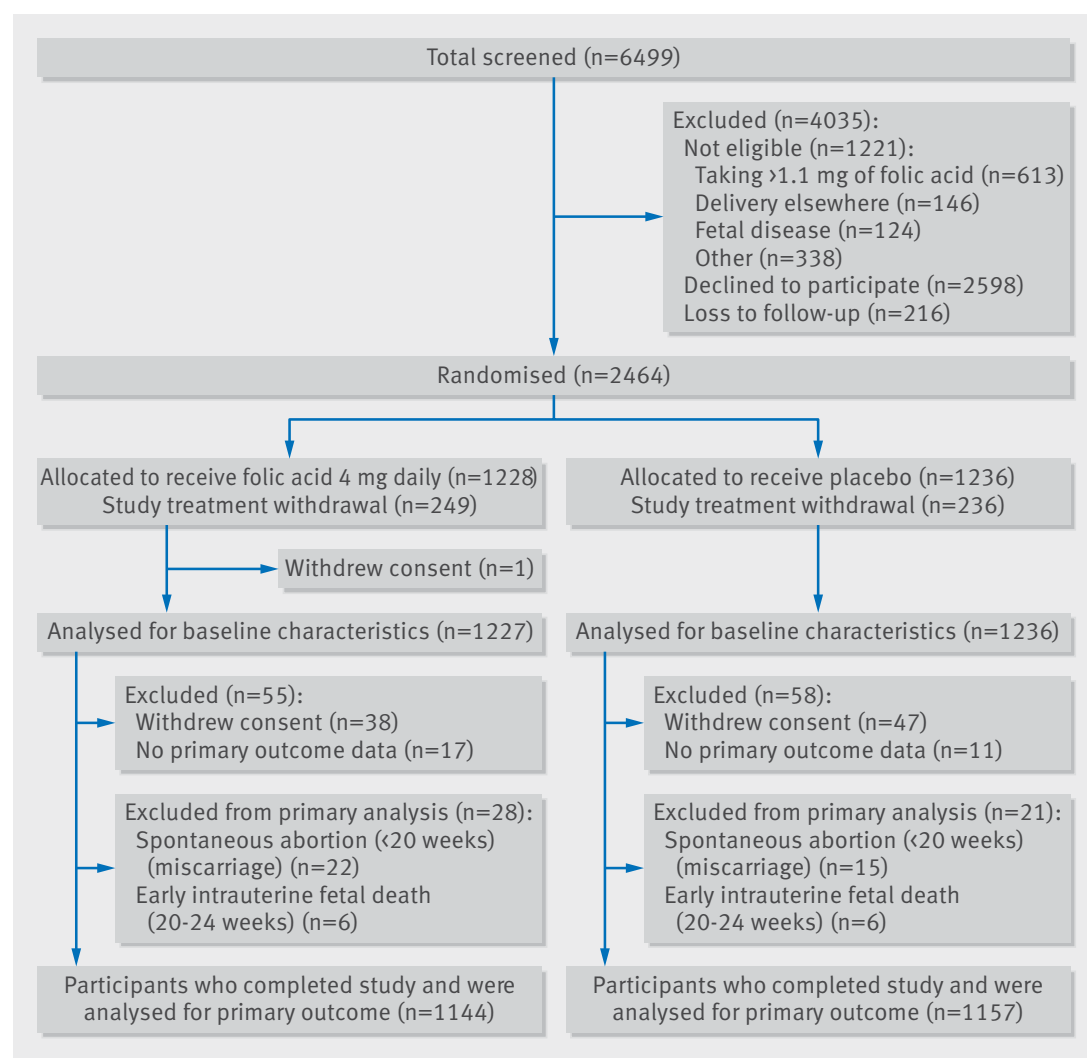

Fig 1 | Trial flow diagram outcome ascertainment $(\mathrm{n}=85)$, primary outcome data were available for 2301 women (93.4\%) (1144 in the folic acid group and 1157 in the placebo group) (fig 1), representing a follow-up rate of $96.5 \%$. A total of 485 women across both groups discontinued study treatment but remained in the trial and provided data for primary and secondary outcomes.

The distribution of baseline and pregnancy characteristics was similar between the two groups. More than $80 \%$ of pregnant women in both groups reported taking supplemental folic acid or folic acid containing vitamins. Among the 1941 (78.8\%) women who returned study treatment bottles, 1465 (75.5\%) took at least $75 \%$ of their pills, confirming a high compliance rate (table 1). Analyses of blood samples from 50 participants (19 in folic acid group and 31 in placebo group) in Canadian centres indicated that serum folate was substantially higher in the folic acid group (mean $260.1 \vee 77.8 \mathrm{nmol} / \mathrm{L}, \mathrm{P}=0.008$ ) and red blood cell folate levels were similar (2700 $v$ $2680 \mathrm{nmol} / \mathrm{L}, \mathrm{P}=0.88$ ).

No statistically significant differences were observed in reported adverse events or severe adverse events between the two groups (see supplementary table S2). The effect did not differ by country (see supplementary table S4).

\section{Discussion}

The results of our international randomised controlled multicentre trial did not show evidence that supplementation with high dose folic acid (4.0-5.1 mg) initiated between eight and 16 completed weeks of gestation and continued until delivery prevents preeclampsia in at risk women. We adjusted analyses for potential confounders by parity, maternal age, and cigarette smoking and confirmed there was no effect of folic acid on the prevention of pre-eclampsia. When we explored the effect of high dose folic acid on risk of pre-eclampsia by country, no difference in effect was observed.

\section{Comparison with other studies}

Supplementation with folic acid during pregnancy is now common in many countries of the world. In our previous cohort study, of 2951 pregnant women recruited between 2002 and 2005 in Ottawa and Kingston, Canada, 2713 (91.9\%) took folic acid supplements during pregnancy, ${ }^{9}$ and of women who used folic acid supplementation, only 544 (20.0\%) discontinued in the third trimester, whereas 447 (16.5\%) used more than $2.0 \mathrm{mg} /$ day. $^{9}$ Similar patterns were observed in our trial population, with more than $80 \%$ of women taking supplemental folic acid. Supplementation with high dose folic acid (usually 4.0-5.0 mg daily) in pregnant women has already become widespread beyond the first trimester. ${ }^{9131425-29}$ Anecdotal evidence suggests that supplementation with high dose folic acid is occurring outside the recommendations for use only in early pregnancy for prevention of neural tube defects, even though the most recent Cochrane review of folic acid in
After excluding women who experienced miscarriage $(\mathrm{n}=37)$, experienced early intrauterine fetal death (2024 weeks of gestation, $n=12$ ), had no primary outcome data available $(n=28)$, or withdrew consent before 
Table 1 | Comparison of maternal baseline and pregnancy characteristics between trial arms. Values are numbers (percentages) unless stated otherwise Characteristics Folic acid group $(n=1227)$ Placebo group $(n=1236)$

Country:

\begin{tabular}{l}
\hline Canada \\
\hline Australia \\
\hline Argentina \\
\hline Jamaica \\
\hline UK \\
\hline
\end{tabular}

\begin{tabular}{|l|}
\hline $600(48.9)$ \\
\hline $157(12.8)$ \\
\hline $61(5.0)$ \\
\hline $29(2.4)$ \\
\hline $380(31.0)$ \\
\hline $308(25.1)$ \\
\hline $203(16.5)$ \\
$84(6.8)$ \\
\hline $98(8.0)$ \\
$233(19.0)$ \\
\hline $606(49.4)$ \\
\hline
\end{tabular}

$607(49.1)$

$153(12.4)$

$61(4.9)$

$32(2.6)$

History of pre-eclampsia

Chronic hypertension

Type 1 diabetes

Type 2 diabetes

Twin pregnancy

Body mass index $\geq 35$

Parity:

0

$413(33.7)$

$383(31.0)$

$303(24.5)$

$241(19.5)$

$72(5.8)$

$84(6.8)$

$229(18.5)$

$656(53.1)$

1

$498(40.6)$

$420(34.0)$

$316(25.7)$

$499(40.4)$

Maternal age (years):

$<20$

$10(0.8)$

20-29

$439(35.8)$

30-34

$411(33.5)$

$\geq 35$

$367(29.9)$

$317(25.6)$

Mean (SD) age (years)

Maternal ethnicity:

Native/Aboriginal

$31(5.4)$

$10(0.8)$

$447(36.2)$

$441(35.7)$

White

$35(2.85)$

Black

$970(79.0)$

Asian

$93(7.6)$

Latino/Hispanic

$45(3.7)$

Indian/South Asian

$31(2.5)$

Declined to answer

$45(3.7)$

$8(0.65)$

$31(5.4)$

Prepregnancy body mass index

$<18.5$

$15(1.2)$

$18.5-<25$

$25-<30$

$230(18.7)$

$24(1.94)$

$30-<35$

$210(17.1)$

$164(13.4)$

$\geq 35$

$607(49.5)$

Mean (SD) prepregnancy body mass index

$34(8.6)$

$987(79.8)$

$107(8.7)$

$50(4.05)$

$22(1.8)$

$36(2.9)$

$10(0.8)$

Education level:

High school and below

College/university not completed

College/university completed

$353(28.8)$

$198(16.15)$

$675(55.1)$

$11(0.9)$

$225(18.2)$

$199(16.1)$

$146(11.8)$

$655(53.0)$

34 (13)

Gestational age (weeks) at recruitment:

8-12

$386(31.5)$

13-16

$841(68.5)$

$348(28.2)$

$197(16.0)$

$689(55.8)$

Mean (SD) gestational age (weeks)

$14(1.9)$

$433(35.0)$

$803(65.0)$

Smoking during pregnancy:

Yes

$98(8.0)$

$1046(85.2)$

$95(7.7)$

Quit during pregnancy

$83(6.8)$

$035(83.7)$

Alcohol intake during pregnancy:

Yes

$23(1.9)$

$106(8.6)$

Quit during pregnancy

Folic acid supplementation*

Supplementation of high dose $(\geq 4.0 \mathrm{mg} / \mathrm{d}$ folic acid at randomisation

$977(80.0)$

$27(2.2)$

$227(18.5)$

$955(77.3)$

$254(20.5)$

$989(80.6)$

$1016(82.2)$

$346(28.2)$

$335(27.1)$

$358(29.2)$

$340(27.5)$

Calcium supplement at randomisation

$97(7.9)$

$109(8.8)$

Mean (SD) dietary folate ( $\mu \mathrm{g})$

Visit 1 (8-16 completed weeks' gestation)

Visit 2 (24-26 completed weeks' gestation)

494 (209), $n=1215$

504 (222), $n=1225$

494 (209), $n=1008$

500 (213), $n=1023$

Compliancet:

$$
\leq 50 \%
$$

$108(11.2)$

$106(10.8)$

$140(14.5)$

$122(12.5)$

$50-<75 \%$

$716(74.3)$

$749(76.7)$

*Includes multivitamin containing folic acid.

tCalculated on returned study treatment $(n=1941)$. Remaining participants did not return any study treatment and compliance could not be calculated $(n=522)$.

Among participants with primary outcome data available $(n=2301), 169(14.8 \%)$ in the folic acid group had a diagnosis of pre-eclampsia compared with $156(13.5 \%)$ in

the placebo group (relative risk $1.10,95 \%$ confidence interval 0.90 to $1.34, \mathrm{P}=0.37$ ). Differences in the rates of HELLP $(1.21,0.37$ to $3.96, \mathrm{P}=0.75)$, severe pre-eclampsia (1.52, 0.81 to 2.84 ,

$\mathrm{P}=0.19$; table 2), and all other maternal outcomes were not statistically significant (table 2). 


\begin{tabular}{|c|c|c|c|c|}
\hline Outcomes & Folic acid group & Placebo group & Relative risk $(95 \% \mathrm{CI})$ & $P$ value \\
\hline Spontaneous abortion (miscarriage) & $27(2.3), n=1172$ & $21(1.8), n=1180$ & 1.29 (0.74 to 2.28$)$ & 0.37 \\
\hline Placental abruption & $12(1.0), n=1169$ & 19 (1.6), n=1179 & $0.64(0.31$ to 1.31$)$ & 0.21 \\
\hline Premature rupture of membranes & 215 (18), $n=1169$ & 224 (19), $n=1180$ & $0.97(0.82$ to 1.15$)$ & 0.71 \\
\hline HELLP syndrome & $6(0.52), n=1144$ & $5(0.43), n=1156$ & $1.21(0.37$ to 3.96$)$ & 0.75 \\
\hline Severe pre-eclampsia & 24 (2.10), $n=1144$ & $16(1.4), n=1156$ & $1.52(0.81$ to 2.84$)$ & 0.19 \\
\hline Antenatal inpatient length of stay (days) & $5.6(7.7)^{\star}, n=221$ & $5.2(6.2)^{\star}, n=232$ & $0.34(7.0)(-0.96$ to 1.63$)+$ & 0.61 \\
\hline \multicolumn{5}{|c|}{$\begin{array}{l}\text { HELLP=haemolysis, elevated liver enzymes, low platelets. } \\
\text { *Mean (SD). } \\
\text { †Mean (SD) difference }(95 \% \mathrm{Cl}) \text {. } \\
\text { A total of } 2738 \text { infants were born to women recruited into the trial (1364 in the folic acid group and } 1374 \text { in the placebo group). The rate of stillbirth } \\
\text { was } 1.1 \% \text { in the folic acid group and } 1.9 \% \text { in the placebo group }(0.60,0.30 \text { to } 1.19 ; \text { table } 3) \text {. No statistically significant differences were observed in } \\
\text { occurrence of adverse neonatal outcomes between the two groups. }\end{array}$} \\
\hline
\end{tabular}

pregnancy for maternal health outcomes was not able to report on pre-eclampsia owing to lack of data from clinical trials. ${ }^{30}$ Caution should always be exercised in recommending treatments before thorough evaluation has been completed, including follow-up of offspring when possible.

\section{Strengths and limitations of this study}

FACT has several notable strengths. Firstly, it was designed to be as conclusive as possible in a rigorous, large, randomised, double blinded, placebo controlled, phase III, international and multicentre trial. The trial conduct adhered to strong research and ethical principles, high data completeness, and compliance by participants. The follow-up rate was greater than $95 \%$ and compliance to the study treatment was greater than $75 \%$ in most of the study population. Steps were taken to ensure the lowest possible risk of bias, although we did not investigate baseline folic acid values, compliance, and levels during pregnancy in subgroups of high risk factors for pre-eclampsia.
A clear advantage of our trial is the robust randomised design, although some limitations are present. Preeclampsia has a complex heterogenous aetiology despite characteristic phenotypic outcomes. ${ }^{31}$ In the original trial protocol we clearly laid out the criteria for the definition of pre-eclampsia and used this for case adjudication. These criteria have remained consistent with NICE guidelines for the diagnosis of pre-eclampsia, although there have been revisions to the definition of pre-eclampsia in other settings, including Canada. ${ }^{32}$ As a result, using the revised guidelines, there may be additional women in the study population who would have a diagnosis of preeclampsia; however, owing to the randomised design this would be balanced across the treatment groups and not anticipated to affect the association between folic acid and pre-eclampsia, regardless of definition. Finally, power was reduced from $90 \%$ to $80 \%$, but because we found no evidence in favour of the study intervention, even an increase of power would have been unlikely to find a treatment effect.

\begin{tabular}{|c|c|c|c|c|}
\hline Outcomes & Folic acid group & Placebo group & Relative risk $(95 \% \mathrm{Cl})^{\star}$ & $P$ value* \\
\hline \multicolumn{5}{|l|}{ Dichotomised outcomes: } \\
\hline Stillbirth & $15(1.1), n=1364$ & 26 (1.9), $n=1374$ & $0.60(0.30$ to 1.19$)$ & 0.14 \\
\hline Intrauterine growth restriction $<3$ rd centile & 20 (1.4), $n=1347$ & 25 (1.9), $n=1348$ & 0.76 (0.41 to 1.39$)$ & 0.37 \\
\hline Intrauterine growth restriction $<10$ th centile & 151 (11.2), $n=1347$ & 144 (11), $n=1348$ & $1.03(0.81$ to 1.30$)$ & 0.82 \\
\hline Neonatal death & $8(0.60), n=1343$ & $11(0.82), n=1347$ & $0.87(0.31$ to 2.44$)$ & 0.79 \\
\hline Perinatal mortality & $23(1.7), n=1364$ & $37(2.7), n=1374$ & $0.63(0.37$ to 1.05$)$ & 0.07 \\
\hline Retinopathy of prematurity & $21(1.6), n=1342$ & $13(0.97), n=1347$ & $1.20(0.54$ to 2.66$)$ & 0.65 \\
\hline Periventricular leukomalacia & $4(0.30), n=1343$ & $2(0.15), n=1347$ & $2.00(0.37$ to 10.92$)$ & 0.42 \\
\hline Early onset sepsis & $3(0.22), n=1342$ & $9(0.67), n=1347$ & 0.34 (0.09 to 1.23$)$ & 0.10 \\
\hline Necrotising enterocolitis & $8(0.60), n=1343$ & $3(0.22), n=1347$ & $2.04(0.49$ to 8.57$)$ & 0.33 \\
\hline Intraventricular haemorrhage & $18(1.3), n=1343$ & $19(1.4), n=1347$ & $0.97(0.47$ to 2.00$)$ & 0.94 \\
\hline Ventilation & $49(3.6), n=1346$ & $30(2.2), n=1348$ & $1.61(0.97$ to 2.66$)$ & 0.06 \\
\hline Need for oxygen at 28 days & $9(0.74), n=1220$ & $3(0.2), n=1227$ & $2.37(0.61$ to 9.14$)$ & 0.21 \\
\hline NICU admission & $299(22), n=1346$ & $267(20), n=1348$ & $1.08(0.91$ to 1.28$)$ & 0.37 \\
\hline $\begin{array}{l}\text { Composite severe adverse fetal or neonatal } \\
\text { outcome }^{\star}\end{array}$ & $63(4.7), n=1349$ & $51(3.8), n=1348$ & 1.20 (0.80 to 1.80$)$ & 0.38 \\
\hline \multicolumn{5}{|l|}{ Continuously distributed outcomes: } \\
\hline NICU length of stay & $16(27)+, n=299$ & $17(23)+, n=263$ & $-1.60(-5.84$ to 2.64$) \ddagger$ & 0.46 \\
\hline $\begin{array}{l}\text { NICU=neonatal intensive care unit. } \\
{ }^{*} \text { Generalised estimating equation was used to accou } \\
\text { included any of retinopathy of prematurity, periventr } \\
\text { ventilation, need for oxygen at } 28 \text { days, and NICU ad } \\
\text { †Mean (SD). } \\
\text { ¥Mean difference }(95 \% \mathrm{CI}) \text {. }\end{array}$ & $\begin{array}{l}\text { correlation between two } \\
\text { eukomalacia, early onse } \\
\text { n. }\end{array}$ & $\begin{array}{l}\text { es or infants from the } \\
\text { sis, necrotising entero }\end{array}$ & $\begin{array}{l}\text { pregnancy. Composite outc } \\
\text { intraventricular haemorrha }\end{array}$ & \\
\hline
\end{tabular}




\section{Conclusion and policy implications}

Future directions for this research include exploration of the increased risk of pre-eclampsia in mothers carrying twins and using high dose folic acid, and the potential protective effect of folic acid on perinatal death warrants ongoing study. Perhaps most importantly, FACT provides a unique opportunity to follow the participants and their offspring to study the effects of high dose folic acid during prenatal development on long term maternal and child health, given the potential epigenetic effects of folic acid. Funding has been obtained to follow these FACT offspring for mortality and neurocognitive development through to six years of age.

Our well powered trial did not find benefit for high dose folic acid supplementation beyond the first trimester for the prevention of pre-eclampsia or related maternal and neonatal adverse outcomes. The trial deals with an important public health issue: the lack of demonstrated benefit of high dose folic acid supplementation beyond the first trimester for women at high risk of developing pre-eclampsia indicates that high dose recommendation should now cease, and the search for an effective and acceptable strategy to prevent pre-eclampsia must continue.

\section{AUTHOR AFFILIATIONS}

On behalf of: the FACT Collaborating Group

${ }^{1}$ OMNI Research Group, Clinical Epidemiology Program, Ottawa Hospital Research Institute, Ottawa, Ontario, Canada

${ }^{2}$ School of Epidemiology, Public Health and Preventive Medicine, University of Ottawa, Ottawa, Ontario, Canada

${ }^{3}$ Department of Obstetrics, Gynecology \& Newborn Care, University of Ottawa, Ottawa, Ontario, Canada

${ }^{4}$ Institute of Cellular Medicine, The Medical School, Newcastle University, Newcastle upon Tyne, UK

${ }^{5}$ Obstetric Medicine, Robinson Research Institute, University of Adelaide, South Australia, Australia

${ }^{6}$ Women's and Children's Hospital, Adelaide, South Australia, Australia

${ }^{7}$ Department of Obstetrics and Gynaecology, Faculty of Medical Sciences, The University of the West Indies, Mona, Kingston, Jamaica

${ }^{8}$ Centro Rosarino de Estudios Perinatales (CREP), Rosario, Santa Fé, Argentina

${ }^{9}$ Queen's Perinatal Research Unit, Kingston General Hospital, Department of Obstetrics and Gynecology, Queens University, Kingston, Ontario, Canada

${ }^{10}$ Mother \& Child Axis, Centre de recherche du Centre Hospitalier Universitaire de Sherbrooke, Sherbrooke, Quebec, Canada

${ }^{11}$ Centre de Recherche, Universite de Sherbrooke, Sherbrooke, Quebec, Canada

${ }^{12}$ Department of Obstetrics and Gynecology, Faculty of Medicine and Health Sciences, Centre de recherche du CHUS, University of Sherbrooke, Sherbrooke, Quebec, Canada;

${ }^{13}$ Cardiovascular Research Methods Centre, University of Ottawa Heart Institute, Ottawa, Ontario, Canada

${ }^{14}$ Department of Medicine, University of Ottawa, Ottawa, Ontario, Canada

${ }^{15}$ Clinical Epidemiology Program, Ottawa Hospital Research Institute, Ottawa, Ontario, Canada

${ }^{16}$ Women and Children's Health Research Institute, University of Alberta, Edmonton, Alberta, Canada

${ }^{17}$ Department of Obstetrics and Gynecology, University of Alberta, Edmonton, Alberta, Canada

${ }^{18}$ Department of Physiology, University of Alberta, Edmonton,
Alberta, Canada

${ }^{19}$ Maternal-Fetal Medicine Division, Department of Obstetrics and Gynecology, Mount Sinai Hospital, University of Toronto, Toronto, Ontario, Canada

${ }^{20}$ Departments of Medicine, School of Epidemiology, Public Health and Preventive Medicine, University of Ottawa, Ottawa, Ontario, Canada

${ }^{21}$ Department of Obstetrics and Gynaecology, Monash University, Clayton, Victoria, Australia

${ }^{22}$ Department of Obstetrics, University Medical Center, Utrecht, Utrecht, Netherlands

${ }^{23}$ Department of Obstetrics, Academic Medical Center, Amsterdam, Netherlands

We thank the participants in FACT, site investigators, research staff at the participating sites, and staff at the Ottawa Hospital Research Institute for their support and hard work. A full list of the FACT Collaborating Group is available in the supplementary file.

Contributors: MCW and SWW designed the trial, planned the analyses, and wrote the manuscript with assistance from RRW, NR, LMG, SR, WH, DSS, GC, GS, WDF, GW, STD, JK, DC, DF, DJC, JC, TR BJM, and MAO. ES with assistance from DJC carried out the statistical analyses. All authors participated in the review and critical revisions of the final manuscript. MCW is the guarantor. The corresponding author attests that all listed authors meet authorship criteria and that no others meeting the criteria have been omitted.

Funding: This study was sponsored by the Ottawa Hospital Research Institute, and funded by the Canadian Institutes of Health Research (grants 198801 and 98030). FACT was conceived, designed, and coordinated independently of the funding source. The funder did not act as sponsor for the trial and had no role in analysis, interpretation of the data, writing of the report, or decision to submit for publication. Competing interests: All authors have completed the ICMJE uniform disclosure form at www.icmje.org/coi disclosure.pdf and declare: no support from any organisations for the submitted work; no financial relationships with any organisations that might have an interest in the submitted work in the previous three years; no other relationships or activities that could appear to have influenced the submitted work.

Ethical approval: FACT was approved by the Ottawa Health Sciences research ethics board (2009107), and at all participating sites. All applicable regulatory agency approvals were also obtained.

Data sharing: The authors of this trial commit to making data available upon reasonable request. Requests for access to data from FACT should be addressed to the corresponding author.

Transparency: The lead author (MCW) affirms that this manuscript is an honest, accurate, and transparent account of the study being reported; that no important aspects of the study have been omitted; and that any discrepancies from the study as planned (and, if relevant, registered) have been explained.

This is an Open Access article distributed in accordance with the Creative Commons Attribution Non Commercial (CC BY-NC 4.0) license, which permits others to distribute, remix, adapt, build upon this work non-commercially, and license their derivative works on different terms, provided the original work is properly cited and the use is noncommercial. See: http://creativecommons.org/licenses/by-nc/4.0/.

1 American College of Obstetricians and Gynecologists, Task Force on Hypertension in Pregnancy. Hypertension in Pregnancy. Obstet Gynecol 2013;122:1122-31. doi:10.1097/01. AOG.0000437382.03963.88

2 Magee LA, Helewa M, Rey E. Hypertension Guideline Committee: Strategic Training Initiative in Research in the Reproductive Health Sciences (STIRRHS) Scholars. Diagnosis, evaluation, and management of the hypertensive disorders of pregnancy. J Obstet Gynaecol Can 2008;30(Suppl):S1-2. http://www.ncbi.nlm.nih.gov/ pubmed/18817592. doi:10.1016/S1701-2163(16)32776-1

3 Duley L. Maternal mortality associated with hypertensive disorders of pregnancy in Africa, Asia, Latin America and the Caribbean. Br I Obstet Gynaecol 1992;99:547-53. http://www.ncbi.nlm.nih.gov/ pubmed/1525093. doi:10.1111/j.1471-0528.1992.tb13818.x

4 Wen SW, Xie R. Maternal mortality. Encyclopedia of Human Biology. 2014.

5 Zhang J, Meikle S, Trumble A. Severe maternal morbidity associated with hypertensive disorders in pregnancy in the United States. Hypertens Pregnancy 2003;22:203-12. doi:10.1081/PRG-120021066

6 Liu A, Wen SW, Bottomley J, Walker MC, Smith G. Utilization of health care services of pregnant women complicated by 
preeclampsia in Ontario. Hypertens Pregnancy 2009;28:76-84. doi:10.1080/10641950802366252

7 Whitfield MF, Grunau RV, Holsti L. Extremely premature (« or = 800 g) schoolchildren: multiple areas of hidden disability. Arch Dis Child Fetal Neonatal Ed 1997;77:F85-90. http://www.ncbi.nlm.nih. gov/pubmed/9377151. doi:10.1136/fn.77.2.F85

8 Hua X, Zhang J, Guo Y, et al. Effect of folic acid supplementation during pregnancy on gestational hypertension/preeclampsia: A systematic review and meta-analysis. Hypertens Pregnancy 2016;35:447-60 doi:10.1080/10641955.2016.1183673

9 Wen SW, Chen X-K, Rodger M, et al. Folic acid supplementation in early second trimester and the risk of preeclampsia. Am J Obstet Gynecol 2008;198:45.e1-7. doi:10.1016/j.ajog.2007.06.067

10 Catov JM, Nohr EA, Bodnar LM, Knudson VK, Olsen SF, Olsen I. Association of periconceptional multivitamin use with reduced risk of preeclampsia among normal-weight women in the Danish National Birth Cohort. Am J Epidemiol 2009;169:1304-11. doi:10.1093/aje/kwp052

11 Li Z, Ye R, Zhang L, Li H, Liu J, Ren A. Folic acid supplementation during early pregnancy and the risk of gestational hypertension and preeclampsia. Hypertension 2013;61:873-9. doi:10.1161/HYPERTENSIONAHA.111.00230

12 Vanderlelie /, Scott R, Shibl R, et al. First trimester multivitamin/ mineral use is associated with reduced risk of pre-eclampsia among overweight and obese women. Matern Child Nutr 2014;12:1-10. doi:10.1111/mcn.12133

13 Wen SW, Guo Y, Rodger M, et al. Folic acid supplementation in pregnancy and the risk of pre-eclampsia-A cohort study. PLoS One 2016;11:e0149818. doi:10.1371/journal.pone.0149818

14 Sayyah-Melli M, Ghorbanihaghjo A, Alizadeh M, Kazemi-Shishvan M, Ghojazadeh M, Bidadi S. The effect of high dose folic acid throughout pregnancy on homocysteine (Hcy) concentration and pre-eclampsia: A randomized clinical trial. PLoS One 2016;11:e0154400. doi:10.1371/journal.pone.0154400.

15 Merchant AT, Msamanga G, Villamor E, et al. Multivitamin supplementation of HIV-positive women during pregnancy reduces hypertension. J Nutr 2005;135:1776-81. doi:10.1093/jn/135.7.1776

16 Saccone G, Sarno L, Roman A, Donadono V, Maruotti GM Martinelli P. 5-Methyl-tetrahydrofolate in prevention of recurrent preeclampsia. J Matern Fetal Neonatal Med 2016;29:916-20. doi:10.3109/14767058.2015.1023189

17 Wen SW, Zhou J, Yang Q, Fraser W, Olatunbosun O, Walker M. Maternal exposure to folic acid antagonists and placentamediated adverse pregnancy outcomes. CMA/ 2008;179:1263-8. doi:10.1503/cmaj.080859

18 Wald N, Sneddon J, Densem J, et al. MRC Vitamin Study Research Group. Prevention of neural tube defects: results of the Medical Research Council Vitamin Study. Lancet 1991;338:131-7. doi:10.1016/0140-6736(91)90133-A

19 Czeizel AE, Dudás I. Prevention of the first occurrence of neural-tube defects by periconceptional vitamin supplementation. N EnglJ Med 1992;327:1832-5. doi:10.1056/NEJM199212243272602.

20 Wilson RD, Wilson RD, Audibert F, et al, Genetics Committee, Special Contributors. Pre-conception Folic Acid and Multivitamin Supplementation for the Primary and Secondary Prevention of Neural Tube Defects and Other Folic Acid-Sensitive Congenital Anomalies. J Obstet Gynaecol Can 2015;37:534-52 doi:10.1016/S1701-2163(15)30230-9.
21 Institute of Obstetricians \& Gynecologists Royal College of Physicians of Ireland. Clinical Practice Guideline: Nutrition for pregnancy. 2016;1.1. https://rcpi-live-cdn.s3.amazonaws.com/wp-content/ uploads/2016/05/22.-Nutrition-during-Pregnancy.pdf

22 Gomes S, Lopes C, Pinto E. Folate and folic acid in the periconceptional period: recommendations from official health organizations in thirty-six countries worldwide and WHO. Public Health Nutr 2016;19:176-89. doi:10.1017/S1368980015000555

23 Walker MC, Finkelstein SA, Rennicks White R, et al. The Ottawa and Kingston (OaK) Birth Cohort: development and achievements. J Obstet Gynaecol Can 2011;33:1124-33. doi:10.1016/S1701-2163(16)35080-0.

24 Duckitt K, Harrington D. Risk factors for pre-eclampsia at antenatal booking: systematic review of controlled studies. BMJ 2005;330:565. doi:10.1136/bmj.38380.674340.EO

25 Tolarova M, Harris J. Reduced recurrence of orofacial clefts after periconceptional supplementation with high-dose folic acid and multivitamins. Teratology 1995;51:71-8. doi:10.1002/ tera.1420510205

26 Czeizel AE, Vereczkey A, Szabó I. Folic acid in pregnant women associated with reduced prevalence of severe congenital heart defects in their children: a national population-based casecontrol study. Eur J Obstet Gynecol Reprod Biol 2015;193:34-9. doi:10.1016/j.ejogrb.2015.06.024

27 Papadopoulou E, Stratakis N, Roumeliotaki T, et al. The effect of high doses of folic acid and iron supplementation in early-to-mid pregnancy on prematurity and fetal growth retardation: the mother-child cohort study in Crete, Greece (Rhea study). Eur J Nutr 2013;52:327-36. doi:10.1007/s00394-012-0339-z.

28 Shahraki AD, Dehkordi NZ, Lotfizadeh M. Comparison of high dose and low dose folic acid supplementation on prevalence, onset and severity of preeclampsia. Adv Biomed Res 2016;5:192. doi:10.4103/2277-9175.190944

29 Hashemi M, Heshmat-Ghahdarijani K, Zarean E, Baktash F, Mortazavi ZS. Evaluation of the effect of high-dose folic acid on endothelial dysfunction in pre-eclamptic patients: A randomized clinical trial. J Res Med Sci 2016;21:114. doi:10.4103/1735-1995.193505

30 Lassi ZSZ, Salam RA, Haider BA, Bhutta ZA. Folic acid supplementation during pregnancy for maternal health and pregnancy outcomes. Cochrane Database Syst Rev 2013:3.CD006896 doi:10.1002/14651858.CD006896. pub2.www.cochranelibrary.com.

31 Uzan J, Carbonnel M, Piconne O, Asmar R, Ayoubi JM. Pre-eclampsia: pathophysiology, diagnosis, and management. Vasc Health Risk Manag 2011;7:467-74 doi:10.2147/VHRM.S20181.

32 Tranquilli AL, Dekker G Magee L et al. The classification, diagnosis and management of the hypertensive disorders of pregnancy: A revised statement from the ISSHP. Pregnancy Hypertens 2014:4:97-104. doi:10.1016/j.preghy.2014.02.001.

Supplementary information: Supplemental appendix with tables S1-S4

Supplementary information: Members of the FACT Collaborating Group 